\title{
Les outils d'évaluation de la biodiversité et des services écosystémiques recommandés aux entreprises : compromis entre crédibilité, pertinence et légitimité
}

Biodiversity valuation tools recommended to business: tradeoffs between credibility, relevance and legitimacy

Anastasia Wolff, Natacha Gondran et Christian Brodhag

\section{(2) OpenEdition}

Édition électronique

URL : https://journals.openedition.org/developpementdurable/11649

DOI : 10.4000/developpementdurable.11649

ISSN : $1772-9971$

Éditeur

Association DD\&T

Référence électronique

Anastasia Wolff, Natacha Gondran et Christian Brodhag, « Les outils d'évaluation de la biodiversité et des services écosystémiques recommandés aux entreprises : compromis entre crédibilité, pertinence et légitimité », Développement durable et territoires [En ligne], Vol. 8, $n^{\circ} 1$ | Avril 2017, mis en ligne le 30 avril 2017, consulté le 15 février 2023. URL : http://journals.openedition.org/developpementdurable/ 11649 ; DOI : https://doi.org/10.4000/developpementdurable.11649

Ce document a été généré automatiquement le 29 septembre 2020

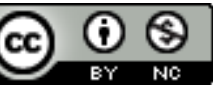

Creative Commons - Attribution - Pas d'Utilisation Commerciale 4.0 International - CC BY-NC 4.0 https://creativecommons.org/licenses/by-nc/4.0/ 


\section{Les outils d'évaluation de la biodiversité et des services}

\section{écosystémiques recommandés aux entreprises : compromis entre crédibilité, pertinence et légitimité}

Biodiversity valuation tools recommended to business: tradeoffs between credibility, relevance and legitimacy

Anastasia Wolff, Natacha Gondran et Christian Brodhag

1 Les activités anthropiques exercent des pressions contribuant à une érosion rapide de la biodiversité et affectant la capacité des écosystèmes à fournir les biens et services dont nous dépendons (Millennium Ecosystem Assessment, 2003; Cardinale et al., 2012). L'évaluation des impacts anthropiques et de l'état de la biodiversité - définie comme la variabilité biologique à l'échelle des gènes, des espèces et des écosystèmes - et des services écosystémiques n'est cependant pas triviale. En effet, les liens fonctionnels entre biodiversité et services écosystémiques sont complexes et encore mal caractérisés (Mace, Norris et Fitter, 2012) et les informations sur l'état biologique des écosystèmes peuvent s'avérer insuffisantes pour comprendre et intégrer dans les décisions les interactions entre fonctionnement des écosystèmes, organisations sociales et systèmes économiques (Laurila-Pant et al., 2015).

2 Parmi les trois grandes catégories d'approches évaluatives (biophysiques, monétaires et basées sur des indicateurs composites) (Gasparatos et Scolobig, 2012), les outils d'évaluation monétaire font l'objet d'une demande sociale grandissante (Milanesi, 2010). Ceci peut être mis en parallèle avec l'hypothèse selon laquelle l'érosion de la biodiversité n'a pu être enrayée jusqu'ici parce que la société ne l'évalue pas à sa juste valeur (Myers et Reichert, 1997; Milanesi, 2010; Suarez et Corson, 2013). Le terme "valeur» est ambivalent et prend des sens contrastés selon les acteurs et les disciplines (Guiral, 2013 ; 
IPBES, 2015). L'attrait qu'exerce l'évaluation monétaire, comme modalité de qualification de la biodiversité, tient notamment au fait qu'elle constitue une synthèse quantitative et unidimensionnelle d'enjeux recoupant diverses échelles et dimensions socio-écologiques, et ce en des termes familiers aux décideurs (KPMG, 2014 ; Sipkens et al., 2014). Certaines études économiques (Costanza et al., 1997; Chevassus-au-Louis, Salles et Pujol, 2009; Hoegh-Guldber, Beal et Chaudhry, 2015) ont ainsi eu de forts retentissements tant dans la sphère scientifique qu'auprès des acteurs publics et privés.

3 Les acteurs économiques et financiers affichent un intérêt croissant pour les outils d'évaluation pour la biodiversité et les services écosystémiques, et s'impliquent dans leur développement (Hanson et al., 2009 ; PUMA, 2012 ; WBCSD, 2013). Ainsi, des organisations telles que le World Business Council for Sustainable Development (WBCSD, 2011), des groupes spécialisés dans le conseil (PwC, 2013) mais aussi des associations environnementales (IBAT, 2015) ou des institutions académiques (Willis et al., 2012) proposent des outils à destination des entreprises. L'attention que portent les entreprises à ces nouveaux outils s'explique par différentes motivations. D'un point de vue stratégique, les entreprises ont besoin d'outils pour mieux appréhender la matérialité des enjeux liés à la biodiversité et aux services écosystémiques, en termes d'impact et de dépendance. Cerner les principaux risques à anticiper dans le cadre de leur devoir de vigilance (due diligence en anglais) est critique pour limiter les risques de réputation (Dempsey, 2013; WBCSD, 2013). L'intégration de ces facteurs de risque par certaines institutions financières laisse présager de possibles répercussions en termes d'accès aux capitaux (Waage et Kester, 2015). De plus, les outils d'évaluation pour la biodiversité et les services écosystémiques peuvent faciliter la comparaison de différents scénarios et contribuer à améliorer l'acceptabilité d'un projet (Dempsey, 2013). Cette évaluation peut aussi être motivée par la volonté d'identifier des opportunités, de capter de nouvelles sources de revenus, via notamment le développement d'instruments de marché (paiements pour services écosystémiques, marchés de compensation écologique) (WBCSD, 2013). D'autre part, les entreprises ont aussi besoin d'outils managériaux pour encadrer la mise en œuvre de leur politique biodiversité, suivre les progrès réalisés (EpE, 2013). Enfin, les outils d'évaluation peuvent appuyer la communication des entreprises auprès de leurs parties prenantes (EpE, 2013). Depuis 2002 et la loi sur les «Nouvelles Régulations économiques", les sociétés françaises cotées en Bourse doivent présenter, dans leur rapport du conseil d'administration ou du directoire, des informations sur «les mesures prises pour préserver ou développer la biodiversité » (décret $n^{\circ}$ 2012-557). Il existe donc une demande pour des méthodologies standardisées.

On observe aujourd'hui un développement et une diversification importants des outils d'évaluation pour la biodiversité et les services écosystémiques (Thievent, 2015 ; Waage et Kester, 2015). Ces outils peuvent avoir des implications variées en termes de représentation et de gestion par l'entreprise de ses liens à la biodiversité et aux services écosystémiques. Ceci soulève d'une part la question des hypothèses sur lesquelles reposent ces outils, qui peuvent être contestables scientifiquement ou socialement du fait $\mathrm{du}$ système de valeurs sur lequel ils s'appuient, et d'autre part la question de leur efficacité en termes de modification des représentations de l'environnement et, in fine, d'une meilleure prise en compte des questions environnementales dans les décisions.

5 Cet article vise trois objectifs concomitants. Le premier est d'expliciter certains partis pris qui découlent du choix, par une entreprise, d'un outil d'évaluation pour la biodiversité et les services écosystémiques. Le second objectif est de mettre en évidence 
l'existence de tensions entre différentes dimensions qui déterminent l'efficacité de ces outils comme aide à la décision : la crédibilité, la pertinence et la légitimité. Enfin, les implications de ces partis pris seront discutées au regard des usages potentiels, par l'entreprise, des résultats produits.

Est présentée ici une évaluation de dix-huit outils d'évaluation de la biodiversité et des services écosystémiques recommandés aux entreprises. Ce travail s'appuie sur une grille d'analyse développée à partir des travaux de Cash et al. (2002) et Sarkki et al. (2014) pour évaluer les outils en fonction de leur crédibilité, pertinence et légitimité. Des compromis existent-ils entre ces trois dimensions ? Sont-ils associés à certains choix techniques des outils? Quelles peuvent être les conséquences de ces arbitrages en fonction de la finalité de l'évaluation pour l'entreprise en termes de gestion de la biodiversité et des services écosystémiques ? Cet article vise à apporter un éclairage à ces questions de recherche.

7 L'article est structuré en quatre sections. La Section 1 présente les systèmes de valeur sous-jacents aux orientations méthodologiques des outils d'évaluation de la biodiversité et des services écosystémiques et met en évidence un déficit d'études portant sur l'efficacité et les effets empiriques des outils. Les concepts de crédibilité, pertinence et légitimité sont retenus comme critères d'évaluation des outils. La Section 2 présente les dix-huit outils sélectionnés pour l'étude et la méthode d'évaluation de leur crédibilité, pertinence et légitimité. Les résultats de l'analyse quantitative sont présentés en Section 3. Les liens observés entre les caractéristiques et la crédibilité, pertinence et légitimité des outils sont discutés dans la Section 4 et mis en perspective avec la finalité d'usage des outils par les entreprises.

\section{Qualification des outils d'évaluation}

\subsection{Méthodes d'évaluation et systèmes de valeurs}

Les outils d'évaluation de la biodiversité et des services écosystémiques sont façonnés par les valeurs des acteurs impliqués dans leur conception, au travers des choix méthodologiques effectués (Vandevelde, 2013). Ils reposent ainsi sur des hypothèses, souvent implicites, et issues de systèmes de valeurs. Un système de valeur peut se définir comme l'ensemble des valeurs auxquelles se réfèrent les individus, les sociétés et les organisations pour réguler leurs comportements (Díaz et al., 2015). Ces hypothèses sousjacentes définissent notamment la perspective biocentrée ou anthropocentrée de l'évaluation ${ }^{1}$, l'adoption d'une approche réductionniste ou systémique, ainsi que l'acceptabilité des compromis faits entre les différents enjeux de durabilité (Gasparatos et Scolobig, 2012). Le concept de service écosystémique s'inscrit ainsi dans une perspective anthropocentrée. En effet, « une fonction écologique ne prend valeur de service à l'homme que dans la mesure où des usages socio-économiques, la réglementation et les valeurs sociales la reconnaissent comme telle et la canalisent de manière à ce qu'elle réponde à des besoins humains, actuels ou anticipés » (Maresca et al., 2011, p. 9).

Bien que les méthodes d'évaluation de la biodiversité puissent être classifiées sur la base de multiples critères (Spurgeon, 2014), Gasparatos et Scolobig proposent de les distinguer selon trois grandes catégories d'approches: biophysiques, monétaires et basés sur des indicateurs composites (Gasparatos et Scolobig, 2012). Les approches biophysiques visent à quantifier des données environnementales biologiques (ex. : richesse spécifique, taille de population) et physiques (ex.: fragmentation des habitats, flux de matières). Les 
approches de monétarisation sont basées sur des modèles de comportements humains et reposent sur l'hypothèse que ce sont les préférences subjectives des individus qui créent la valeur. La valeur de la biodiversité et des services écosystémiques est traduite en unité monétaire, impliquant leur substituabilité avec d'autres capitaux, et ignorant généralement de nombreux services non quantifiés, voire quantifiables (Gadrey, 2011). L'approche monétaire s'inscrit ainsi dans une conception dite «faible» de la durabilité. Enfin, les approches à base d'indicateurs composites impliquent de nombreux choix méthodologiques. Les phases de sélection d'indicateurs, pondération, normalisation et agrégation cachent souvent, sous des choix apparemment méthodologiques et techniques, des prises de position en termes de visions, valeurs et priorités. Ainsi, les choix, et donc les résultats de l'évaluation, dépendent des experts construisant l'outil d'évaluation.

10 Afin de répondre aux préoccupations concrètes des décideurs, les méthodes d'évaluation pour la biodiversité et les services écosystémiques sont opérationnalisées via le développement d'outils spécifiques s'appuyant sur une palette méthodologique plus ou moins large et fournissant un ou plusieurs types de résultats (biophysiques, monétaires, basés sur des indicateurs composites).

\subsection{Questionner l'engouement pour l'évaluation de la biodiversité et des services écosystémiques}

11 "On ne gère bien que ce que l'on mesure ». Cet adage, omniprésent dans les discours managériaux, est réemployé à l'égard de la conservation de la biodiversité et des ressources naturelles. Cette idée se retrouve dans certains discours affirmant que la mauvaise gestion de la biodiversité et des services écosystémiques vient du fait qu'« on ne protège pas ce à quoi on ne donne pas de valeur » (Myers et Reichert, 1997, p. xix). Dans cette perspective, certains acteurs soutiennent que la monétarisation de la biodiversité et des services écosystémiques permet de mieux les intégrer aux décisions stratégiques et à la gestion des activités en traduisant les enjeux en des termes familiers aux décideurs (Bräuer, 2003 ; Polasky, 2008 ; TEEB, 2012).

Peu de travaux de recherche semblent s'être penchés sur les usages réels et les apports pratiques des évaluations de la biodiversité et des services écosystémiques, soit du fait que les chercheurs accordent peu d'attention à cet enjeu, soit du fait d'une sousutilisation de ces méthodologies (Laurans et al., 2013). Le rapport Eco4Biz du WBCSD (WBCSD, 2013) et l'analyse comparative réalisée par la mission "Économie de la biodiversité » de la Caisse des Dépôts et Consignations, matérialisée par l'outil GoBIODIV+ (Thievent, 2015) discutent l'intérêt d'une palette d'outils pour les entreprises en illustrant leur argumentaire par quelques retours d'expérience. Bagstad et al. ont comparé et appliqué différents outils pour évaluer les services écosystémiques du bassin de San Pedro (Bagstad et al., 2013). Au-delà de ces rares initiatives, l'analyse critique de ces nouveaux outils et des changements dans les modalités de gestion de la biodiversité et des services écosystémiques qu'ils engendrent semble peu développée au regard des enjeux qu'ils représentent. Waage et Kester soulignent ainsi que « peu, voire aucun, de ces outils n'a fait l'objet d'un processus de vérification et de validation robuste, transparent et indépendant, en particulier en termes d'application au secteur privé » (Waage et Kester, 2015, p. 12). L'intérêt affiché des outils d'évaluation de la biodiversité et des services écosystémiques, destinés 
aux entreprises, parait davantage reposer sur des présupposés relatifs aux vertus de l'évaluation que sur une validation par une analyse des effets empiriques.

\subsection{Crédibilité, pertinence et légitimité des interfaces connaissances/décisions}

13 Les outils d'évaluation de la biodiversité et des services écosystémiques peuvent être envisagés comme des interfaces articulant des connaissances pour générer des informations en support à la prise de décision. La crédibilité, la pertinence et la légitimité ont été proposées comme des attributs de l'efficacité des interfaces science-décision (Cash et al., 2002 ; IPBES, 2014 ; Rodela et al., 2015). La crédibilité de l'information est relative à la perception par un acteur de sa qualité, de sa validité et de sa robustesse scientifique. L'évaluation de la crédibilité est à la fois fonction de la confiance dans le processus de production de l'information et dans les institutions qui apportent les connaissances (Sarkki et al., 2014). La pertinence fait référence au fait que l'évaluation est appropriée pour informer le choix du décideur. Enfin, la légitimité reflète le sentiment que le processus d'évaluation a pris en compte la diversité des systèmes de valeurs et de croyances des parties prenantes, et a été conduit de façon impartiale et juste dans son traitement des opinions et intérêts divergents (Cash et al., 2002).

14 Ces trois attributs semblent présenter des complémentarités et des compromis (Cash et al. , 2002; Sarkki et al., 2014). Il peut ainsi être difficile de maximiser simultanément la crédibilité, la pertinence et la légitimité d'un outil d'évaluation. Il n'est cependant pas trivial d'analyser les synergies et divergences de ces trois dimensions, car il n'existe pas de mesure directe de la crédibilité, la pertinence et la légitimité (CPL). Heink et al. soulignent le caractère subjectif de ces trois qualités, qui peuvent avoir des significations variables en fonction des contextes et des acteurs (Heink et al., 2015). Dans l'optique de mobiliser ces attributs pour évaluer l'efficacité d'une interface science-décision, Heink et al. recommandent d'expliciter les liens entre les attributs CPL et l'objet d'étude, de spécifier précisément ce que recouvrent ces concepts pour les rendre opérationnels et transparents et d'adapter les critères d'évaluation aux définitions des attributs CPL retenus.

15 S'appuyant sur un cadre d'analyse construit autour de ces trois dimensions - crédibilité, pertinence, légitimité -, la suite de l'article présente une analyse quantitative portant sur un échantillon de dix-huit outils recommandés aux entreprises.

\section{Matériels et méthodes}

\subsection{Présentation des outils}

\subsubsection{Définition de l'échantillon d'outils}

Relativement peu d'entreprises communiquent sur les outils qu'elles utilisent en interne, ou qui sont mobilisés pour produire les résultats diffusés vers l'extérieur. Ne pouvant ainsi identifier les principaux outils d'évaluation de la biodiversité et des services écosystémiques utilisés, nous nous concentrerons sur les outils faisant l'objet d'une communication récurrente dans des publications conseillant les entreprises sur les moyens à mettre en œuvre pour mieux appréhender les enjeux de biodiversité. 
17 Une revue de la littérature grise a permis d'identifier cinq rapports, principalement à destination des acteurs économiques et financiers, préconisant des outils d'évaluation de la biodiversité et des services écosystémiques (Oregon State University, Sustainable Northwest et University of Oregon, 2013 ; WBCSD, 2013 ; KPMG, 2014 ; Maxwell, McKenzie et Traldi, 2014 ; Waage et Kester, 2015). L'analyse croisée de ces documents a permis d'identifier pas moins de cent vingt-neuf outils d'évaluation différents traitant entre autres ou exclusivement de la biodiversité et des services écosystémiques. La majorité des outils n'étaient cités que dans un seul document et aucun n'était présenté par l'ensemble des cinq rapports.

Nous n'avons retenu que les outils cités dans au moins trois rapports différents, soit dixhuit outils au total, présentés dans le tableau 1. La mention d'une utilisation par des entreprises a pu être documentée pour quatorze outils.

\begin{tabular}{|c|c|c|c|c|c|}
\hline Acronyme & Outil, URL, référence & Développeurs et sponsors & $\begin{array}{l}\text { Annee de } \\
\text { lancement }\end{array}$ & $\begin{array}{l}\text { Rapports } \\
\text { citant } \\
\text { l'outil }\end{array}$ & $\begin{array}{l}\text { Exemples d'entreprises ayant } \\
\text { utilisé I'outil }\end{array}$ \\
\hline ARIES & $\begin{array}{l}\text { Artificial Intelligence for Ecosystem services, } \\
\text { http://www.ariesonline.org (Villa et al, 2014) }\end{array}$ & $\begin{array}{l}\text { UNEP-WCMC, US NSF, Basque Centre } \\
\text { for Climate Change, University yof } \\
\text { Vermont, Conservatition Interational, } \\
\text { Earth Economics, Instituto di Ecologia } \\
\text { INECOL }\end{array}$ & $\begin{array}{l}\text { portail } \\
\text { fonctionnel } \\
\text { en } 2012\end{array}$ & $(1,3,4,5)$ & $?$ \\
\hline BROA & $\begin{array}{l}\text { Biodiversity Risk \& Opportunity Assessment, } \\
\text { http:/ www. batbiodiversity. org BROA }\end{array}$ & $\begin{array}{l}\text { British American Tobacco Biodiversity } \\
\text { Partnership }\end{array}$ & 2006 & $(1,3,5)$ & $\begin{array}{l}\text { British American Tobacco, India } \\
\text { Tobacco Company }\end{array}$ \\
\hline CEV & $\begin{array}{l}\text { Guide to Corporate Ecosystem Valuation, } \\
\text { hutp:///wwwwwwbcsd. org work-program ecosystems cev.aspx } \\
\text { (WBCSD, 2011) }\end{array}$ & $\begin{array}{l}\text { WBCSD, IUCN, World Resources } \\
\text { Institute (WRI), ERM, PwC }\end{array}$ & 2011 & $(1,3,4,5)$ & $\begin{array}{l}\text { 14, ex: AkzoNobel, Eni, } \\
\text { Holcim, Lafarge, Mondi }\end{array}$ \\
\hline Costing & $\begin{array}{l}\text { Costing Nature, http://www.policysupport.org costingnature } \\
\text { (Mulligan et a1, 2010) }\end{array}$ & $\begin{array}{l}\text { King's College London, AmbioTEK, } \\
\text { UNEP-WCMC }\end{array}$ & 2009 & $(1,3,5)$ & $?$ \\
\hline Eco-LCA & $\begin{array}{l}\text { Ecologically Based Life-Cycle Assessment, } \\
\text { www. resilience.osueduecococa (Zhang, Baral et Balsshi, 2010) }\end{array}$ & Ohio State University & 2009 & $(1,3,5)$ & Cook Composites and Polymers \\
\hline EP\&L & 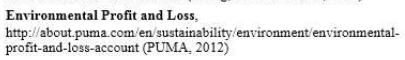 & Puma (Kering), Trucost, PwC & 2010 & $(1,2,3)$ & PUMA/Kering \\
\hline ESB & $\begin{array}{l}\text { NVI Toolkit - Ecosystem Services Benchmark, } \\
\text { http:///wwww.naturalvalueinitiative.org content } 003 \text { 3 } 303 \text {. php (Grigg et } \\
\text { al., 2009) }\end{array}$ & The Natural Value Initiative & 2009 & $(1,3,4)$ & $\begin{array}{l}\text { +31 entreprises, ex : Danone, } \\
\text { Nestle, Unilever, British } \\
\text { American Tobacco, Coca-Cola }\end{array}$ \\
\hline ESR & 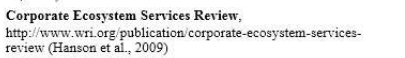 & WRI, WBCSD, Meridian Institute & 2008 & $(1,3,4,5)$ & $\begin{array}{l}\text { De l'ordre de } 300 \text { entreprises, } \\
\text { ex : AdzonNobel, Mondi, } \\
\text { Syngenta, Rio Tinto }\end{array}$ \\
\hline ESR for IA & $\begin{array}{l}\text { Ecosystem Serrices Review for Impact Assessment, } \\
\text { http-//wwww.wri.org publication ecosystem-services-review-impact- } \\
\text { assessment (Landsberg et al, 2013) }\end{array}$ & WRI & 2011 & $(1,3,5)$ & $\begin{array}{l}\text { Testé pour des projets miniers, } \\
\text { agro-alimentaires et d'energie } \\
\text { éolienne (entreprises anonymes) }\end{array}$ \\
\hline LEFT & 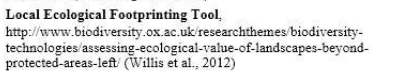 & $\begin{array}{l}\text { Biodiversity Institute University of } \\
\text { Oxford, Department of Computer Science } \\
\text { University of Oxford, Statoil }\end{array}$ & 2012 & $(1,3,5)$ & Statoil \\
\hline IBAT & $\begin{array}{l}\text { Integrated Biodiversity Assessment Tool, } \\
\text { https://wwww:batfortusiness. org (Dempsey, 2013) }\end{array}$ & $\begin{array}{l}\text { BirdLifie International, Conservation } \\
\text { International, IUCN, UNEP-WCMC }\end{array}$ & 2005 & $(3,4,5)$ & $\begin{array}{l}\text { +35, ex : Bank of America, BP, } \\
\text { Cargill, JPMorgan Chase, Total }\end{array}$ \\
\hline InVEST & $\begin{array}{l}\text { Integrated Valuation of Environmental Services and Tradeoffs, } \\
\text { http:///www naturalcapitalproject.org InVEST.hthl (Tallis et } \\
\text { Polasky, 2009) }\end{array}$ & Natural Capital Project & 2006 & $(1,3,4,5)$ & Lafarge, Dow Chemical \\
\hline MIMES & $\begin{array}{l}\text { Multi-scale Integrated Models of Ecosystem Services, } \\
\text { http } / \text { www. afordablefutures.com/ (Boumans et al, 2015) }\end{array}$ & AFORDable Futures ILC & 2007 & $(1,3,4,5)$ & ? \\
\hline NSv & $\begin{array}{l}\text { NatureServe Vista, http://www.natureserve.org conservation. } \\
\text { tools natureserve--vista }\end{array}$ & NatureServe & 2004 & $(1,3,5)$ & Entreprises anonymes \\
\hline SERVES & $\begin{array}{l}\text { Ecosystem Valuation Toolkit - Simple Effective Resource for } \\
\text { Valuing Ecosystem Service, http://esvaluation org about-serves/ }\end{array}$ & Earth Economics & $\begin{array}{l}\text { ouverture du } \\
\text { portail en } \\
2012\end{array}$ & $(1,3,4,5)$ & Entreprises anonymes \\
\hline TESSA & 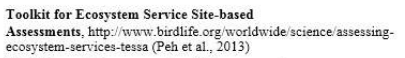 & $\begin{array}{l}\text { Cambridge Conservation Initiative et } \\
\text { Birdlife International }\end{array}$ & 2011 & $(1,3,5)$ & ? \\
\hline TIMM & $\begin{array}{l}\text { Total Impact Measurement and Management, } \\
\text { http:/www. pwc.com totalimpact (PwC, 2013) }\end{array}$ & $\mathrm{PwC}_{\mathrm{C}} \mathrm{er}$ & 2013 & $(1,2,3)$ & $\begin{array}{l}\text { Clients de PwC, ex: Scottish } \\
\text { Hydro Electric }\end{array}$ \\
\hline TrueP & True Price, http:/trueprice.org services/ service-true-price/ & True Price Foundation & $\begin{array}{l}\text { en cours de } \\
\text { develop- } \\
\text { pement }\end{array}$ & $(1,2,3)$ & $\begin{array}{l}\text { HIVOS, AzkoNobel, Tony's } \\
\text { Choocolonely, Royal BAM Group }\end{array}$ \\
\hline
\end{tabular}

\subsubsection{Description des caractéristiques}

19 À partir de la documentation disponible en libre accès sur internet (sites officiels des outils, journaux scientifiques...), nous avons dans un premier temps dégagé certaines caractéristiques $^{2}$ des outils sélectionnés, synthétisées dans le Tableau 2. 


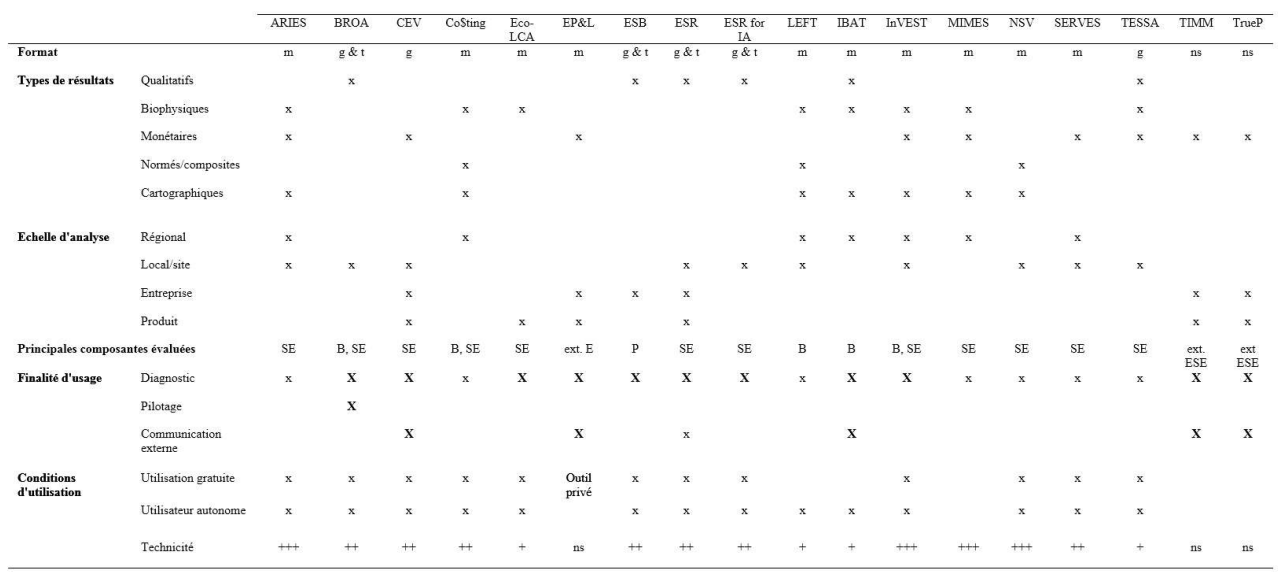

20 Ces outils présentent des caractéristiques variées. Différentes catégories d'acteurs sont impliquées dans leur conception: des institutions académiques (ex.: University of Vermont, King's College London, Ohio State University...), des organisations non gouvernementales (ONG) spécialisées dans l'environnement (ex.: UICN, BirdLife International, Conservation International...), des organismes institutionnels (ex. : UNEPWCMC, la National Science Foundation (NSF) américaine), des entreprises (cabinets de conseil, tels que PwC ou ERM, coalitions d'entreprises, WBCSD par exemple, ou encore entreprises individuelles, telles que Puma). Sept des dix-huit outils étudiés ont été développés par des entreprises (seules ou en partenariat avec d'autres acteurs). Concernant leurs conditions d'utilisation, douze sont gratuits et quatorze peuvent être utilisés de façon autonome par les usagers. A contrario, EP\&L (PUMA, 2012), TIMM et True Price sont des outils payants dont le déploiement est opéré par les tenants de la propriété intellectuelle.

21 Ces outils revêtent différents formats. Les modèles informatiques, permettant des analyses quantitatives, tiennent une place prépondérante. Six outils se présentent sous forme de guides éventuellement accompagnés d'un fichier Excel aidant l'utilisateur dans le traitement des données. Ces guides apportent des éléments de vulgarisation et d'opérationnalisation des concepts associés à la biodiversité et aux services écosystémiques, donnent des instructions, précisent les procédures pour conduire les évaluations, donnent des exemples d'applications, de bonnes pratiques, de retours d'expérience.

Parmi les quatorze outils qui proposent une approche quantitative, neuf produisent des résultats monétarisés, huit fournissent des indicateurs biophysiques, trois produisent des indicateurs composites normalisés (sans unité). Les outils basés sur des approches monétaires ne sont pas l'apanage des entreprises, l'outil d'évaluation monétaire SERVES par exemple a été développé par l'ONG Earth Economics, et inversement des entreprises ont été impliquées dans le développement des outils non monétaires BROA, ESR (Hanson et al., 2009), ESB (Grigg et al., 2009) et LEFT (Willis et al., 2012).

La moitié des outils étudiés autorisent des évaluations à plusieurs échelles (du produit à l'échelle régionale/globale). L'échelle locale est la plus récurrente (10 outils sur 18).

L'effort initial à fournir par l'utilisateur pour collecter et intégrer les données d'entrée nécessaires varie considérablement. Ainsi, l'outil LEFT requiert uniquement de rentrer les coordonnées géographiques du site d'intérêt et génère automatiquement un rapport rendant compte de sa valeur écologique à partir de bases de données préexistantes. Les outils ARIES (Villa et al., 2014), MIMES (Boumans et al., 2015) et NSV nécessitent quant à 
eux d'entrer un nombre important de données pour construire des scénarios géospatialisés.

En termes de finalités d'usage, l'ensemble de ces outils se donne comme objectif d'aider l'entreprise à établir un diagnostic dans le cadre d'un usage interne, pour appréhender la matérialité des enjeux, supporter la prise de décision lors de l'évaluation de projets, évaluer les performances environnementales. Pour six outils, la documentation associée fait explicitement référence à un usage externe, dans le cadre du reporting RSE ou de la communication à destination des parties prenantes. Un seul outil (BROA) prétend apporter un support au pilotage et à la mise en œuvre des plans d'action.

\subsection{Méthode d'évaluation de la crédibilité, pertinence et légitimité des outils d'évaluation}

\subsubsection{Modèle Crédibilité-Pertinence-Légitimité (CPL)} caractéristiques de la crédibilité, pertinence et légitimité identifiées dans les travaux de (Sarkki et al., 2014, p. 198). La caractéristique « consensus » proposée par Sarkki et al. pour qualifier la légitimité a cependant été remplacée dans notre cadre d'analyse par le critère "portage multi-institutionnel».

\begin{tabular}{|c|c|c|c|}
\hline Qualités Phases & Développement de l'outil & $\begin{array}{l}\text { Processus } \\
\text { d'évaluation }\end{array}$ & Résultats \\
\hline Crédibilité & $\begin{array}{l}\mathrm{C} 1 \text { : Dans le prolongement des } \\
\text { connaissances scientifiques }\end{array}$ & $\begin{array}{l}\text { C2 : Examen de la } \\
\text { qualité }\end{array}$ & $\begin{array}{l}\text { C3 : Explicitation des } \\
\text { incertitudes et des } \\
\text { limites }\end{array}$ \\
\hline Pertinence & $\begin{array}{l}\text { P1 : En réponse aux attentes des } \\
\text { décideurs }\end{array}$ & $\begin{array}{l}\text { P2: Délais } \\
\text { d'évaluation }\end{array}$ & $\begin{array}{l}\text { P3 : Simplicité des } \\
\text { messages }\end{array}$ \\
\hline Légitimité & L1 : Portage multi-institutionnel & $\begin{array}{l}\text { L2 : Inclusion des } \\
\text { parties prenantes }\end{array}$ & $\begin{array}{l}\text { L3 : Représentation } \\
\text { équilibrée des points de } \\
\text { vue }\end{array}$ \\
\hline
\end{tabular}




\subsubsection{Attribution des scores} critère a été spécifié par une assertion pour laquelle un score a été attribué sur une échelle ordinale à trois niveaux : 0 si l'assertion est invalidée, 1 si elle est partiellement validée, 2 si elle est validée. Le choix d'opter pour une échelle à seulement trois niveaux et de définir les critères d'évaluation de façon restrictive a été motivé par la volonté de standardiser l'évaluation et de limiter la part de subjectivité imputable à l'évaluateur.

31 outils a été utilisée pour noter les assertions.

\begin{tabular}{|c|c|c|}
\hline Critères & Définition & Scores $\{0,1,2\}$ \\
\hline \multicolumn{3}{|l|}{ Crédibilité } \\
\hline $\begin{array}{l}\text { C1: Dans le prolongement } \\
\text { des connaissances } \\
\text { scientifiques }\end{array}$ & $\begin{array}{l}\text { L'outil mobilise des concepts, méthodes et données } \\
\text { établis scientifiquement }\end{array}$ & $\begin{array}{l}0: \text { ne mobilise pas de méthodes et données tirées de travaux scientifiques, } 1 \text { : } \\
\text { intermédiaire, } 2: \text { mobilisation de concepts, méthodes et données établis } \\
\text { scientifiquement }\end{array}$ \\
\hline $\mathrm{C} 2$ : Examen de la qualité & $\begin{array}{l}\text { La méthode d'évaluation a fait l'objet d'une revue par } \\
\text { la communauté scientifique }\end{array}$ & $\begin{array}{l}0: \text { pas d'examen, } 1 \text { : déclaratif (note stipulant qu'un comité d'experts a examiné } \\
\text { l'outil), } 2 \text { : avis publiés ou publication dans des revues scientifiques à comité de } \\
\text { lecture }\end{array}$ \\
\hline $\begin{array}{l}\text { C3 : Explicitation des } \\
\text { incertitudes et des limites }\end{array}$ & $\begin{array}{l}\text { La documentation détaille les limites méthodologiques } \\
\text { et les incertitudes des résultats sont spécifiées }\end{array}$ & 0 : méthode non détaillée, 1 : intermédiaire, $2:$ limites et incertitudes spécifíes \\
\hline \multicolumn{3}{|l|}{ Pertinence } \\
\hline $\begin{array}{l}\text { P1: En réponse aux attentes } \\
\text { des décideurs }\end{array}$ & $\begin{array}{l}\text { L'outil répond à une question opérationnelle ou } \\
\text { stratégique }\end{array}$ & $\begin{array}{l}0: \text { outil générique ou non orienté vers les problématiques d'entreprise, } 1 \text { : } \\
\text { intermédiaire, } 2 \text { : outil répondant à une question opérationnelle ou stratégique } \\
\text { spécifique }\end{array}$ \\
\hline P2 : Délais d'évaluation & $\begin{array}{l}\text { Le temps requis pour l'évaluation et la réactualisation } \\
\text { est compatible avec les délais de décisions }\end{array}$ & $\begin{array}{l}0 \text { : de l'ordre de plusieurs mois de collecte et d'analyse des données, } 1 \text { : } \\
\text { intermédiaire, } 2 \text { : faible effort de collecte et d'analyse, de l'ordre de quelques } \\
\text { jours }\end{array}$ \\
\hline P3: Simplicité des messages & $\begin{array}{l}\text { Les résultats sont traduits en des termes vulgarisés, } \\
\text { compréhensibles pour les acteurs économiques et } \\
\text { compatibles avec les systemes de gestion }\end{array}$ & $\begin{array}{l}0: \text { : résultats peu synthétisés et exprimés en termes techniques, } 1: \text { intermédiaire, } 2 \\
\text { : résultats synthétiques exprimés dans des unités communues pour des non } \\
\text { spécialistes et pouvant être intégrés dans les systèmes d'information et de } \\
\text { management (comptables, environnementaux...) d'une entreprise }\end{array}$ \\
\hline \multicolumn{3}{|r|}{ ( } \\
\hline $\begin{array}{l}\text { L1 : Portage multi- } \\
\text { institutionnel }\end{array}$ & $\begin{array}{l}\text { Institutions publiques, ONG environnementales et } \\
\text { acteurs économiques sont associés dans le } \\
\text { développement et la promotion de l'outil }\end{array}$ & $\begin{array}{l}0: \text { un seul type d'organisation, } 1 \text { : deux types d'organisations, } 2 \text { : institutions } \\
\text { publiques, ONG environnementales et acteurs économiques sont associés }\end{array}$ \\
\hline L2 : Large participation & $\begin{array}{l}\text { L'outil invite à l'implication d'une variété de parties } \\
\text { prenantes pour alimenter les inputs de l'évaluation }\end{array}$ & $\begin{array}{l}0: \text { les données d'entrée ou hypothèses ne peuvent pas être orientées par les } \\
\text { parties prenantes, } 1 \text { : possibilité d'impliquer les parties prenantes mentionnée, } 2 \text { : } \\
\text { la structure de l'outil encourage ou implique l'engagenent des parties prenantes }\end{array}$ \\
\hline $\begin{array}{l}\text { L3 : Représentation } \\
\text { équilibrée des points de vue }\end{array}$ & $\begin{array}{l}\text { Le traitement des intérêts des parties prenantes est } \\
\text { équilibré dans les outputs de l'évaluation }\end{array}$ & $\begin{array}{l}0: \text { les résultats explicitent uniquement les intérêts de l'utilisateur, } 1 \text { : } \\
\text { intermédiaire, } 2 \text { : les intérêts de l'ensemble des parties prenantes sont représentées } \\
\text { sur le même plan }\end{array}$ \\
\hline
\end{tabular}

\subsubsection{Analyses statistiques}

Les analyses statistiques ont été réalisées à l'aide du logiciel $\mathrm{R}$ (version 3.1.2). Les corrélations entre les neuf critères CPL ont été analysées avec le test non paramétrique de corrélation de Spearman (les variables étant ordinales) à partir des scores obtenus pour les dix-huit outils. La significativité des coefficients de Spearman a été testée par un test bilatéral. Les corrélations étaient considérées comme significatives pour une $\mathrm{p}$-value inférieure à 0,05 . Le package FactoMineR a été utilisé pour réaliser une analyse en composantes principales (ACP) des dix-huit outils en prenant comme variables les scores des neuf critères CPL. Le type de résultats produits par les outils (qualitatif, non monétaire, monétaire ou combinant monétaire et non monétaire), la présence d'entreprises parmi les concepteurs des outils, ainsi que la finalité d'usage des outils (cf. Tableaux 1 et 2) ont été utilisés comme variables qualitatives illustratives. Les variables illustratives n'influent pas sur le calcul des composantes principales. Elles apportent cependant un éclairage à l'interprétation des variabilités observées en testant a posteriori l'association entre composantes principales et variables illustratives. 


\section{Résultats}

\subsection{Scores CPL}

concernant la pertinence, la simplicité des messages (P2) est positivement associée à l'objectif de répondre à des questions opérationnelles ou stratégiques (P3) ;

concernant la crédibilité, l'examen de la qualité (C2) est positivement corrélé d'une part au développement de l'outil dans le prolongement des connaissances scientifiques (C1), et d'autre part à la communication des incertitudes et des limites (C3).

Une corrélation positive mais relativement faible $(0,48 ;$ p.value $=0,04)$ est observée entre deux critères caractérisant des attributs différents : le traitement équilibré des points de vue $\begin{gathered}(\mathrm{L} 3) \\ \text { Crédibilité }\end{gathered}$ et l'examen $\begin{gathered}\text { de } \\ \text { Pertinence }\end{gathered}$ la qualité $\begin{gathered}\text { (C2). } \\ \text { Légitimité }\end{gathered}$

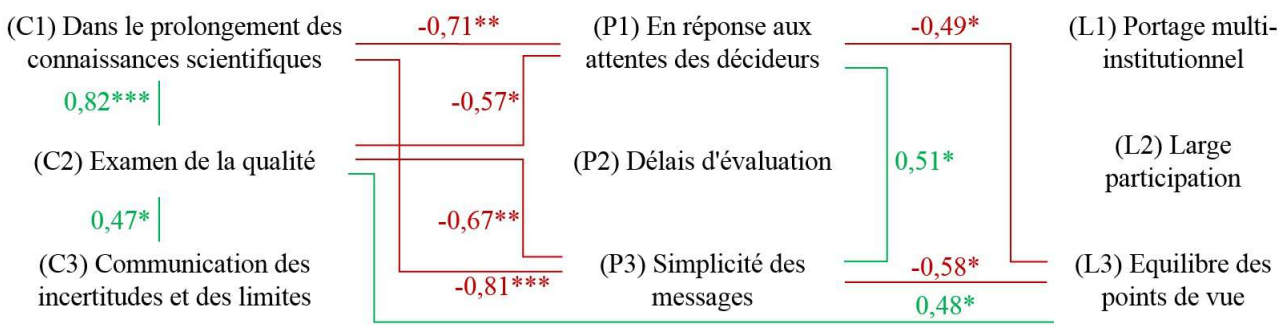

\subsection{Des profils CPL contrastés}

L'ACP des scores assignés aux dix-huit outils pour les neuf critères CPL permet de mettre en évidence des profils d'outils contrastés (figure 2a). Le premier axe principal (qui résume $40,65 \%$ de la variance totale) permet de ségréguer les outils ayant obtenu des 
scores relativement élevés par rapport à leur ancrage scientifique (C1) et l'examen de la qualité méthodologique (C2) (à droite, Dim 1 positive), des outils exposant des messages simples (P3) en réponse aux attentes des décideurs (P1) (à gauche, Dim 1 négative). La contribution respective des variables au premier axe principal est la suivante : $\mathrm{C} 1=24 \%$, $\mathrm{C} 2=19 \%, \mathrm{P} 3=19 \%, \mathrm{P} 1=17 \%$.

Le deuxième axe principal (qui résume 19,56\% de la variance totale) oppose les outils nécessitant une large implication des parties prenantes dans le processus d'évaluation (L2) (Dim 2 positive) et ceux permettant une évaluation rapide (P2) (Dim 2 négative) (contributions respectives des variables au deuxième axe principal : $\mathrm{L} 2=41 \%, \mathrm{P} 2=34 \%$ ).
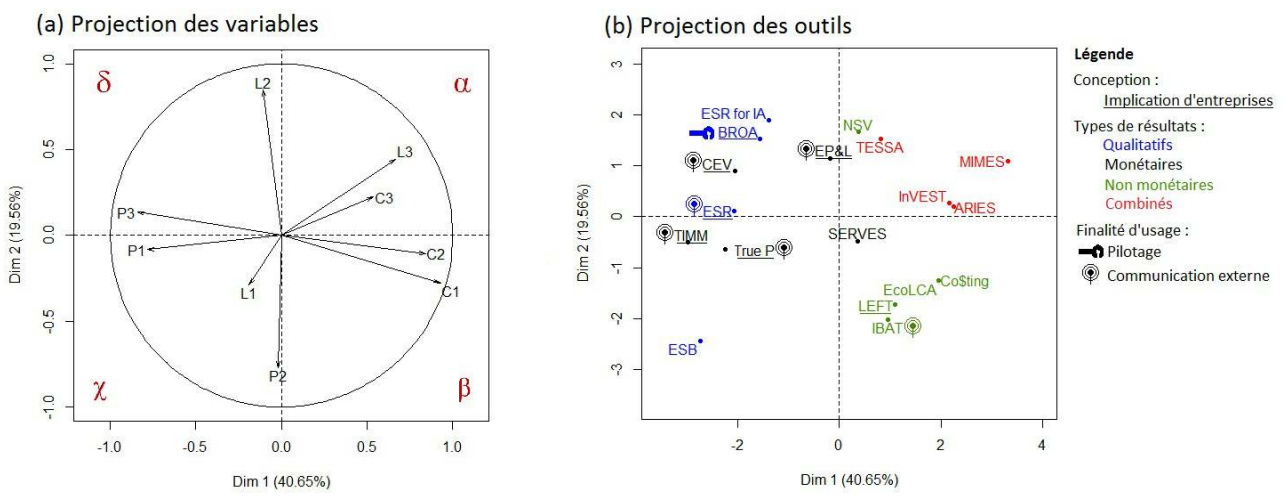

Les deux premiers axes de l'ACP, permettent ainsi de distinguer quatre profils d'outils (figure 2):

(’) des outils relativement crédibles et légitimes mais moins pertinents en vue de l'aide à la décision (ex. : TESSA et InVEST) ;

( $\beta$ ) des outils permettant une évaluation rapide (P2) et crédible ( 1, C2), moins performants en termes d'implication des participants (L2) (ex. : IBAT et LEFT) ;

$(\mathrm{X})$ des outils pertinents pour les décideurs ( $\mathrm{P} 1, \mathrm{P} 2, \mathrm{P} 3)$ mais pouvant présenter des écueils sur le plan de leur légitimité et crédibilité (ex. : ESB);

( $\delta$ ) des outils présentant des messages simples (P3), en réponse aux attentes des décideurs (P1) et intégrant les parties prenantes dans le processus d'évaluation (L2), mais moins performants en termes de crédibilité (ex. : BROA).

\subsection{Profils CPL et caractéristiques des outils}

41 Les trois caractéristiques des outils mobilisées comme variables illustratives sont significativement associées au premier axe principal à savoir :

- le type de résultats produits par les outils $(\mathrm{R} 2=0,78$; p.value $<0,001)$;

- l'implication d'entreprises dans la conception de l'outil ( $\mathrm{R} 2=0,55$; p.value $<0,001)$;

la finalité d'usage $(\mathrm{R} 2=0,37 ; \mathrm{p}$.value $=0,03)$.

Aucune de ces caractéristiques n'est significativement associée au second axe principal.

Ainsi, lorsque des entreprises ont pris part au développement, les outils présentent des scores relativement plus élevés en termes de simplicité du message et d'orientation vers les besoins des décideurs, mais semblent en moyenne moins performants en termes de crédibilité et de présentation de divers points de vue (Dim $1=-1,6$; p.value $<0,001$ ), et inversement pour les outils n'ayant pas impliqué d'entreprise (Dim $1=1,3$; p.value < $0,001)$. 

mais a vocation à informer et rationaliser des choix (Salles, 2011 ; Laurans et al., 2013). Les allégations, concernant les bénéfices pour une entreprise, de conduire une évaluation de la biodiversité et des services écosystémiques dont elle dépend ou qu'elle impacte sont multiples: meilleure gestion des ressources, des risques, amélioration de la communication avec les parties prenantes... L'efficacité de ces outils d'évaluation pour éclairer les choix en entreprise et améliorer la gestion des impacts et de l'état de la biodiversité et des services écosystémiques est cependant, au stade actuel, largement hypothétique. leur développement, d'approches méthodologiques, de niveaux de technicité et de finalités d'usage. Notre étude, qui se limite à l'analyse des caractéristiques des outils en dehors de contextes d'application spécifiques, suggère des arbitrages entre la crédibilité, la pertinence et la légitimité des outils, trois attributs clés pour articuler efficacement connaissances et décisions (Cash et al., 2002). Ceci interpelle quant à la capacité de ces outils à améliorer la gestion effective de la biodiversité et des services écosystémiques. Après avoir exposé les limites de cette étude, nous nous proposons dans cette section de discuter les associations observées entre les profils CPL et les caractéristiques des outils, puis les implications possibles quant aux usages de ces outils par les entreprises.

\subsection{Limites de l'étude} regard des limites de notre étude.

Tout d'abord, notre cadre d'analyse a été défini afin de mettre en évidence de potentielles tensions ou synergies entre crédibilité, pertinence et légitimité des outils. Il n'a pas pour intention de leur attribuer un score qui refléterait leur qualité absolue. Ce cadre d'analyse restreint les dimensions que peuvent recouper les concepts de crédibilité, pertinence et légitimité à des assertions opérationnelles qui ne traduisent que partiellement la substance de ces dimensions. 
D'autre part, les scores CPL ont été évalués sur la base de la documentation accessible sur les sites dédiés aux outils avec un possible risque d'omission d'informations au cours de la recherche et l'analyse documentaires. Afin de limiter la part de subjectivité inhérente à l'évaluateur lors de l'attribution des scores aux outils, la grille de notation a été standardisée et l'échelle de notation réduite à trois niveaux. Lors de l'exercice de notation, il est cependant apparu que certains critères étaient plus objectifs (par exemple le critère L1 portant sur la diversité des organisations impliquées dans le développement de l'outil) que d'autres (par exemple le critère L3 relatif à la présentation équilibrée des intérêts des parties prenantes).

51 De plus, la crédibilité, la pertinence et la légitimité des outils n'ont été évaluées qu'en tenant compte des caractéristiques des outils hors contexte spécifique d'utilisation. La durée de réalisation d'une évaluation (P2), par exemple, a ainsi été notée sur la base des informations mentionnées par les développeurs et des retours d'expérience publiés. Or celle-ci peut varier significativement en fonction du périmètre de l'entreprise, de la disponibilité des données et du nombre d'acteurs impliqués dans l'évaluation. Certains outils (tels que LEFT, ESB ou IBAT) autorisent peu de marge d'adaptation méthodologique alors que d'autres offrent une flexibilité importante (par exemple ARIES, ESR ou CEV). Pour ces derniers, les processus d'utilisation ont potentiellement un fort impact sur la crédibilité, pertinence et légitimité des résultats et décisions issus de l'évaluation.

\subsection{Des arbitrages entre crédibilité, pertinence et légitimité}

L'analyse des scores CPL des dix-huit outils a mis en évidence l'existence d'arbitrages entre crédibilité, pertinence et légitimité. Ces résultats supportent l'hypothèse qu'il existe des compromis, mais éventuellement aussi des synergies entre ces trois dimensions comme l'ont suggéré plusieurs auteurs (Cash et al., 2002; Sarkki et al., 2014). Nous pouvons constater des disparités entre les tensions proposées par Sarkki et al. et celles qui émergent de notre étude. Par exemple, leur analyse corrobore l'existence de tensions entre l'objectif de répondre aux questions opérationnelles des décideurs (P1) versus une approche dans le prolongement des connaissances scientifiques (C1), mais Sarkki et al. n'identifient pas de compromis entre la simplicité des messages (P3) versus une approche dans le prolongement des connaissances scientifiques ( $\mathrm{C} 1$ ) alors que la corrélation négative observée dans notre étude est particulièrement significative.

Les résultats de la présente étude suggèrent que les arbitrages entre crédibilité, pertinence et légitimité pourraient être tributaires :

- de l'implication d'acteurs économiques lors de la conception de l'outil ;

54 - du type de résultats produits (qualitatif, monétaire, non monétaire, ou combinant des valeurs monétaires et non monétaires);

et de la finalité d'usage (diagnostic, pilotage, communication externe).

Concernant les types de résultats produits, les outils combinant valeurs monétaires et non monétaires, tous développés avec des organismes académiques, retranscrivent la complexité des enjeux. Ils requièrent un niveau de technicité avancé et sont relativement lourds à déployer. La priorité est mise sur l'exhaustivité et le réalisme de la modélisation au détriment du caractère opérationnel de l'outil. Les outils monétaires visent à délivrer une réponse synthétique aux questions des décideurs. S'ils apparaissent comme pertinents pour les décideurs, cela semble être aux dépens de leur crédibilité scientifique 
et de la représentation équilibrée des points de vue. Les outils biophysiques et basés sur des indicateurs normés sont les plus rapides à déployer et bénéficient d'une crédibilité scientifique relativement élevée. Restreignant le contexte d'étude aux enjeux techniques, ils semblent peu intégrer les parties prenantes dans le processus d'évaluation. Enfin, les outils qualitatifs ressortent comme globalement pertinents pour répondre aux questions des décideurs et certains mettent l'accent sur la prise en compte des parties prenantes. Néanmoins, ceux-ci peuvent demander un effort de déploiement conséquent et le formalisme de la démarche peut être incertain. Ainsi, le choix d'un outil doit être avant tout cohérent avec l'objectif de l'exercice.

\subsection{Usages et finalités des outils par les entreprises} d'un diagnostic (cliché analytique momentané pour informer la décision en interne), le pilotage (approche procédurale, plans d'action pour assurer un suivi dans le temps) et la communication externe (reporting, justification des arbitrages).

\subsubsection{Communication externe}

Dans l'optique d'une communication externe, les exigences en termes de crédibilité et de légitimité de l'évaluation pourraient être supérieures. L'entreprise s'expose en effet à ce que les résultats soient contestés par ses parties prenantes. Pourtant, notre analyse suggère que les outils promus pour la communication externe sont des outils qui mettent davantage l'accent sur la pertinence pour les décideurs que sur la légitimité et la crédibilité de l'évaluation. La crédibilité scientifique et la représentation équilibrée des points de vue sont les deux critères pour lesquels l'écart avec les outils limités au diagnostic est le plus important. Privilégier trop fortement la dimension pratique d'un outil au détriment de sa robustesse méthodologique et de la légitimité de l'évaluation peut être contre-productif dans le cadre d'une communication externe des résultats. L'écueil le plus récurrent de ces outils concerne l'explicitation de la méthode d'évaluation, à l'exception des outils EP\&L et IBAT pour lesquels la méthode est clairement exposée. Très peu d'éléments sont notamment fournis par les outils TIMM et TRUE $P$, développés par des organismes privés. Ce déficit de transparence méthodologique peut éventuellement être motivé par des problématiques de confidentialité, mais risque de générer auprès des parties prenantes l'impression que les résultats sont issus d'une boîte noire qui ne permet pas de discuter les choix méthodologiques. Quant aux guides ESR et CEV qui laissent une marge de manœuvre conséquente à l'utilisateur, la crédibilité et la légitimité de l'évaluation peuvent être largement influencées par la conduite de l'évaluation. Il appartient donc aux entreprises d'expliciter la démarche suivie afin d'asseoir la crédibilité et la légitimité des informations communiquées.

\subsubsection{Focus sur quatre outils}

Parmi les cinq outils ayant les plus hauts scores CPL totaux, quatre ont effectivement été utilisés par des entreprises (BROA, IBAT, InVEST et LEFT). Nous discutons plus spécifiquement les résultats obtenus pour ces quatre outils et leurs utilisations potentielles.

Développement durable et territoires, Vol. 8, n | Avril 2017 

seul outil qui prétend apporter, au-delà du diagnostic, un support opérationnel en termes de pilotage et de déploiement de plans d'action. Il se distingue par sa dimension pragmatique, avec un focus sectoriel sur l'agriculture et une approche risque/ opportunité nécessitant une implication forte des parties prenantes du terrain. Cet outil est ressorti de l'analyse comme particulièrement performant sur le plan de la légitimité et de la pertinence pour les décideurs, mais apparaît comme moins robuste sur le plan de la crédibilité scientifique. Cet apparent déficit de crédibilité, reflétant la structure de la grille d'évaluation CPL, est cependant à nuancer, car l'outil a fait l'objet d'une revue indépendante par plusieurs organismes bénéficiant d'une solide légitimité (notamment UNEP-WCMC et WWF-UK).

60

\section{l'UNEP-WCMC) et LEFT (développé par le Biodiversity Institute University of Oxford,} Department of Computer Science University of Oxford et Statoil) partagent des caractéristiques et des profils CPL relativement similaires. Les deux outils permettent de géo-localiser les sites importants pour la biodiversité à proximité d'une zone d'intérêt. Leur principale finalité est de faciliter la comparaison de zones d'implantation potentielles et d'anticiper les enjeux écologiques à partir de la spécification d'un minimum de données par l'utilisateur, à savoir les coordonnées géographiques de la zone d'étude. IBAT et LEFT facilitent ainsi le recoupement de diverses sources d'information et diminuent le coût de transaction potentiellement élevé de la collecte de données écologiques pour établir un diagnostic de premier niveau. Les choix méthodologiques pour apprécier l'intérêt écologique des sites sont cependant très différents : IBAT se base sur une approche institutionnelle (sites faisant l'objet d'une protection réglementaire, liste rouge de l'UICN...), alors que LEFT se base sur une approche écologique (caractéristiques locales de fragmentation, vulnérabilité, connectivité, nombre d'espèces par taxon... dérivées notamment de la Global Biodiversity Information Facility). IBAT et LEFT ont obtenu des scores CPL quasiment identiques, à savoir une crédibilité scientifique et une pertinence pour les décideurs élevées, mais un niveau de légitimité moindre, notamment en termes d'implication des parties prenantes. En contexte réel d'utilisation par les entreprises, il peut cependant être envisageable qu'une entreprise engage des processus de concertation en complément de l'évaluation fournie par ces outils. La granulosité de ces outils, qui restent actuellement macroscopiques, invite en effet à apporter un éclairage supplémentaire plus proche du terrain.

61 Enfin, InVEST (développé par le Natural Capital Project) est le seul des quatre outils à ne pas avoir été pensé spécifiquement pour les entreprises et son niveau de technicité est plus élevé. Il nécessite ainsi un investissement relativement lourd de l'entreprise (bien que la segmentation en tiers de l'outil autorise une gradation de cet investissement en fonction des besoins de l'utilisateur). Ce type d'outil est a priori plutôt utilisé pour comparer des scénarios de gestion dans le cas de projets ponctuels particulièrement sensibles. Les nombreuses allégations quant aux finalités d'usages possibles de l'outil (Natural Capital Project, 2015) nous semblent davantage refléter la multi-dimensionnalité que le caractère opérationnel de l'outil qui permet, sur la base d'une quinzaine de modèles, une évaluation spatialisée des services écosystémiques, de leur utilisation et des compromis, exprimés en unités biophysiques et/ou monétaires. Les résultats CPL obtenus par InVEST, à savoir des scores de crédibilité élevés, mais une pertinence pour les décideurs moindre, soulignent la dimension encore très académique de l'outil. 


\subsection{Perspectives de recherche} relations entre les caractéristiques des outils d'évaluation, leur qualité sur le plan de la crédibilité, de la pertinence et de la légitimité, et leurs finalités d'usage. La valeur ajoutée pratique de ces outils n'a pu encore être démontrée de façon tangible. Cet examen est d'autant plus difficile que les comparaisons entre outils sont délicates. Cette difficulté découle de différences importantes dans la définition des services écosystémiques, la structure analytique des outils et le type de résultats produits (Waage, Hwang et Armstrong, 2011). Il s'agit d'un pan de l'évaluation environnementale encore relativement récent qui nécessite d'être examiné plus finement afin de dépasser le caractère réducteur d'un discours présentant l'évaluation comme une panacée. L'analyse des usages concrets des outils d'évaluation par les entreprises et de leurs impacts sur les modalités de gestion de la biodiversité et des services écosystémiques nous semble être une perspective de recherche qui pourrait donner lieu à une enquête par entretiens auprès d'entreprises déclarant utiliser les outils d'évaluation présentés dans cet article : comment s'approprient-elles ces outils? Dans quelle mesure l'outil, le processus d'évaluation et les modalités de communication conditionnent-ils respectivement la crédibilité, pertinence et légitimité des résultats? Quelles sont les actions concrètes mises en place suite à l'évaluation?

\section{Conclusion}

Il existe aujourd'hui une demande pour des méthodes standardisées d'évaluation des impacts et dépendances des entreprises vis-à-vis de la biodiversité et des services écosystémiques. Cependant, les allégations quant aux vertus des outils d'évaluation pour la biodiversité et les services écosystémiques demeurent largement spéculatives. Les apports concrets de ces outils en termes d'évolution des modalités de gestion de la biodiversité et des services écosystémiques restent à démontrer.

Différents systèmes de valeurs et rationalités s'expriment au travers des orientations méthodologiques de ces outils. La biodiversité et les services écosystémiques recouvrent des dimensions variées qui ne peuvent être exhaustivement intégrées dans un même outil. Malgré les simplifications inéluctables, les outils d'évaluation sont donc intrinsèquement complexes de par leur objet d'étude.

L'analyse de dix-huit outils recommandés aux entreprises suggère des tensions structurelles entre leur pertinence pour les décideurs (simplicité des messages, objectif de répondre à des questions opérationnelles ou stratégiques) versus leur crédibilité scientifique (examen de la qualité, développement de l'outil dans le prolongement des connaissances scientifiques) et leur légitimité sociale. Les arbitrages entre crédibilité, pertinence et légitimité semblent dépendants à la fois de l'implication d'acteurs économiques lors de la conception de l'outil, du type de résultats produit par l'évaluation et de la finalité d'usage de l'outil.

66 Ces outils doivent être considérés comme des initiatives d'acteurs ayant leurs propres motivations et rationalités. Leur institutionnalisation constitue, pour les acteurs impliqués dans leur conception et leur diffusion, un levier pour influer sur les 
représentations de la biodiversité. Dans un contexte où diverses organisations poussent au développement de ces approches évaluatives (COP10 CDB, 2010; Spurgeon, 2014; KPMG, 2014), notre grille d'analyse "crédibilité, pertinence, légitimité » propose de questionner certaines stratégies et certains rapports de force à l'œuvre dans ces outils. Des perspectives de recherche pourraient viser à faire le lien entre les caractéristiques des outils utilisés et leur efficacité en termes d'évolution des modalités de gestion de la biodiversité et des services écosystémiques. Le rôle de plus en plus prégnant accordé aux entreprises dans la conservation de la biodiversité (COP10, 2010) appelle en effet à poser un regard critique sur les dynamiques à l'œuvre pour s'assurer qu'elles contribuent à orienter les acteurs vers un modèle plus durable.

\section{BIBLIOGRAPHIE}

Bagstad K.J., Semmens D.J., Waage S., Winthrop R., 2013, « A comparative assessment of decisionsupport tools for ecosystem services quantification and valuation ", Ecosystem Services, vol. 5, p. 27-39.

Boumans R., Roman J., Altman I., Kaufman L., 2015, « The Multiscale Integrated Model of Ecosystem Services (MIMES) : Simulating the interactions of coupled human and natural systems ", Ecosystem Services, vol. 12, p. 30-41.

Bräuer I., 2003, « Money as an indicator : to make use of economic evaluation for biodiversity conservation », Agriculture, Ecosystems \& Environment, vol. 98, n¹-3, p. 483-491.

Cardinale B.J., Duffy J.E., Gonzalez A., Hooper D.U., Perrings C., Venail P., Narwani A., Mace G.M., Tilman D., Wardle D.A., Kinzig A.P., Daily G.C., Loreau M., Grace J.B., Larigauderie A., Srivastava D.S., Naeem S., 2012, « Biodiversity loss and its impact on humanity », Nature, vol. 486, $\mathrm{n}^{\circ} 7401$, p. 59-67.

Cash D.W., Clark W.C., Alcock F., Dickson N.M., Eckley N., Jäger J., 2002, « Salience, credibility, legitimacy and boundaries : Linking research, assessment and decision making », KSG Working Papers Series, RWP02-046, 25 p.

Chevassus-au-Louis B., Salles J.-M., Pujol J.-L., 2009, Approche économique de la biodiversité et des services liés aux écosystèmes - Contribution à la décision publique, La Documentation française (Rapports et documents), $376 \mathrm{p}$.

COP10 CDB, 2010, « Decision X/21. Business engagement », Nagoya, Japan, Convention pour la diversité biologique.

Costanza R., d'Arge R., de Groot R.S., Farber S., Grasso M., Hannon B., Limburg K., Naeem S., O'Neill R.V., Paruelo J., Raskin R.G., Sutton P., van den Belt M., 1997, « The value of the world's ecosystem services and natural capital ", Nature, vol. 387, n6630, p. 253-260.

Dempsey J., 2013, « Biodiversity loss as material risk : Tracking the changing meanings and materialities of biodiversity conservation », Geoforum, vol. 45, p. 41-51.

Díaz S., et al., 2015, « The IPBES Conceptual Framework - connecting nature and people », Current Opinion in Environmental Sustainability, vol. 14, p. 1-16. 
EpE, 2013, Mesurer et piloter la biodiversité, Paris, Entreprises pour l'environnement, 48 p.

Gadrey J., 2011, Sur la valeur économique des chauves-souris comme pesticides, http://alternativeseconomiques.fr/blogs/gadrey/2011/04/22/sur-la-valeur-economique-des-chauves-souriscomme-pesticides/, consulté le 25/06/2015.

Gasparatos A., Scolobig A., 2012, « Choosing the most appropriate sustainability assessment tool », Ecological Economics, vol. 80, p. 1-7.

Grigg A., Cullen, Z., Foxall, J., Strumpf, R., 2009, Linking shareholder and natural value. Managing biodiversity and ecosystem services risk in companies with an agricultural supply chain, Fauna \& Flora International, UNEP Finance Initiative, Fundação Getulio Vargas, 78 p.

Guiral C., 2013, Les valeurs de la biodiversité : un regard sur les approches et le positionnement des acteurs, Paris, FRB, Série expertise et synthèse, $53 \mathrm{p}$.

Hanson C., Ranganathan J., Iceland C., Finisdore J., 2009, The Corporate Ecosystem Services Review. Guidelines for Identifying Business Risks and Opportunities Arising from Ecosystem Change, WRI, Meridian Institute, WBCSD, $48 \mathrm{p}$.

Heink U., Marquard E., Heubach K., Jax K., Kugel C., Neßhöver C., Neumann R.K., Paulsch A., Tilch S., Timaeus J., Vandewalle M., 2015, « Conceptualizing credibility, relevance and legitimacy for evaluating the effectiveness of science-policy interfaces: Challenges and opportunities ", Science and Public Policy, vol. 42, n5, p. 676-689.

Hoegh-Guldber O., Beal D., Chaudhry T., 2015, Reviving the Ocean Economy : the case for action, Suisse, Genève, WWF International, $60 \mathrm{p}$.

IBAT, 2015, IBAT for Business fact sheet, BirdLife International, Conservation International, IUCN, UNEP-WCMC, 2 p.

IPBES, 2014, « Set of principles and procedures guiding the work of IPBES », http:// www.ipbes.net/index.php/11-ipbes/news/news-centre/189-ipbes-principles-procedures, consulté le 04/02/2016.

IPBES, 2015, « Preliminary guide regarding diverse conceptualization of multiple values of nature and its benefits, including biodiversity and ecosystem functions and services (deliverable $3(d)$ ) », IPBES/4/INF/13, IPBES.

KPMG, 2014, A New Vision of Value : Connecting corporate and societal value creation, Pays-Bas, KPMG International, $116 \mathrm{p}$.

Landsberg F., Treweek J., Stickler M., Henninger N., Venn O., 2013, Weaving Ecosystem Services into Impact Assessment. A Step-By-Step Method, Version 1.0, WRI, 46 p.

Laurans Y., Rankovic A., Billé R., Pirard R., Mermet L., 2013, « Use of ecosystem services economic valuation for decision making : Questioning a literature blindspot ", Journal of Environmental Management, vol. 119, p. 208-219.

Laurila-Pant M., Lehikoinen A., Uusitalo L., Venesjärvi R., 2015, « How to value biodiversity in environmental management?», Ecological Indicators, vol. 55, p. 1-11.

Mace G.M., Norris K., Fitter A.H., 2012, « Biodiversity and ecosystem services : a multilayered relationship », Trends in Ecology \& Evolution, vol. 27, n¹, p. 19-26.

Maresca B., Mordret X., Ughetto A.L., Blancher P., 2011, «Évaluation des services rendus par les écosystèmes en France ", Développement durable et territoires, vol. 2, n 3, https:// developpementdurable.revues.org/9053. 
Maxwell D., McKenzie E., Traldi R., 2014, « Valuing natural capital in business. Taking stock : existing initiatives and applications », Natural Capital Coalition, $38 \mathrm{p}$.

Milanesi J., 2010, « Éthique et évaluation monétaire de l'environnement : la nature est-elle soluble dans l'utilité ?», Vertigo, vol. 10, n², http://vertigo.revues.org/10050.

Millennium Ecosystem Assessment, 2003, Ecosystems and human well-being : a framework for assessment, Washington, D.C., USA, Island Press, 266 p.

Mulligan M., Guerry A., Arkema K., Bagstad K.J., Villa F., 2010, « Capturing and quantifying the flow of ecosystem services ", in Silvestri S., Kershaw, F. (Eds.), Framing the Flow : Innovative Approaches to Understand, Protect and Value Ecosystem Services Across Linked Habitat, Cambridge, UK, UNEP, WCMC, p. 26-33.

Myers J.P., Reichert J.S., 1997, « Perspectives on nature's services », in Daily G. (dir.), Nature's services : societal dependence on natural ecosystems, Washington D.C., Island Press, p. xvii-xx.

Nations Unies, 1992, Convention sur la diversité biologique, Rio, Brésil.

Natural capital project, 2015, http://www.naturalcapitalproject.org/pubs/ Web_BusinessBrochure.pdf, consulté le 17/04/2015.

Oregon State University, Sustainable Northwest, University of Oregon, 2013, Payments for Ecosystem Services : Catalog of Online Tools and Resources, $7 \mathrm{p}$.

Peh K.S.-H. et al., « TESSA: A toolkit for rapid assessment of ecosystem services at sites of biodiversity conservation importance », Ecosystem Services, vol. 5, p. 51-57.

Polasky S., 2008, « Valuing Nature : Biophysical or Monetary Measures », Contribution to the meeting Economics and Conservation in the Tropics : A Strategic Dialogue, San Francisco, Jan 31-Feb 1st 2008.

PUMA, 2012, PUMA's Environmental Profit and Loss Account for the year ended 31 December 2010, PUMA, $34 \mathrm{p}$.

PwC, 2013, Measuring and managing total impact : A new language for business decisions, PwC, 48 p.

Rodela R., Reinecke S., Bregt A., Kilham E., Lapeyre R., 2015, « Challenges to and opportunities for biodiversity science-policy interfaces ", Environmental Science \& Policy, vol. 54, p. 483-486.

Salles J.-M., 2011, «Valuing biodiversity and ecosystem services : Why put economic values on Nature? ", Comptes rendus biologies, vol. 334, n5-6, p. 469-482.

Sarkki S., Niemela J., Tinch R., Hove S. Van den, Watt A., Young J., 2014, « Balancing credibility, relevance and legitimacy : A critical assessment of trade-offs in science-policy interfaces », Science and Public Policy, vol. 41, n², p. 194-206.

Sipkens A., de Groot B., Drost R., Aerts M-C., van der Laan R., Midgley L., Beeren D., de Groot Ruiz A., Grosscurt C., 2014, The Business Case for True Pricing Why you will benefit from measuring, monetizing and improving your impact, Consultation draft, Deloitte, EY, PwC, True Price, 50 p.

Spurgeon J., 2014, Natural Capital Accounting for Business : Guide to selecting an approach. Final report to the EU Business and Biodiversity Platform, EU Business@Biodiversity Platform, 41 p.

Suarez D., Corson C., 2013, « Seizing Center Stage : Ecosystem Services, Live, at the Convention on Biological Diversity! », Human Geography, vol. 6, n¹, p. 64-79.

Tallis H., Polasky S., 2009, « Mapping and Valuing Ecosystem Services as an Approach for Conservation and Natural-Resource Management », Annals of the New York Academy of Sciences, vol. $1162, \mathrm{n}^{\circ} 1$, p. 265-283. 
TEEB, 2012, The Economics of Ecosystems and Biodiversity in Business and Enterprise, Earthscan, London and New York, Joshua Bishop, 217 p.

Thievent P., 2015, « Entreprises et biodiversité : quels outils pour quelles décisions? Analyse comparative et guide opérationnel pour l'action », Les Cahiers de BIODIV'2050, 7, Paris, France, Mission Économie de la biodiversité. CDC Biodiversité, 66 p.

Vandevelde J.-C., 2013, « Les choix de tracé des grandes infrastructures de transport : quelle place pour la biodiversité ? ", Développement durable et territoires, vol. $4, \mathrm{n}^{\circ} 1$, https:// developpementdurable.revues.org/9721.

Villa F., Bagstad K.J., Voigt B., Johnson G.W., Portela R., Honzák M., Batker D., 2014, « A

Methodology for Adaptable and Robust Ecosystem Services Assessment », PLoS ONE, vol. 9, n³, p. e91001.

Waage S., Hwang L., Armstrong K., 2011, New Business Decision-Making Aids in an Era of Complexity, Scrutiny, and Uncertainty, BSR, $40 \mathrm{p}$.

Waage S., Kester C., 2015, Making the Invisible Visible : Analytical Tools for Assessing Business Impacts \& Dependencies Upon Ecosystem Services, BSR, 52 p.

WBCSD, 2011, Guide to Corporate Ecosystem Valuation, World Business Council for Sustainable Development, $76 \mathrm{p}$.

WBCSD, 2013, Eco4Biz, Ecosystem services and biodiversity tools to support business decision-making, World Business Council for Sustainable Development, $48 \mathrm{p}$.

Willis K.J. et al., Weissenberger J., 2012, « Determining the ecological value of landscapes beyond protected areas ", Biological Conservation, vol. 147, $\mathrm{n}^{\circ} 1$, p. 3-12.

Zhang Y., Baral A., Bakshi B.R., 2010, « Accounting for ecosystem services in Life Cycle Assessment, Part II : toward an ecologically based LCA », Environmental Science \& Technology, vol. $44, \mathrm{n}^{\circ} 7$, p. 2624-2631.

\section{NOTES}

1. Ces deux perspectives sont traduites par l'Intergovernmental Science-Policy Platform on Biodiversity and Ecosystem Services (IPBES) par la mise en regard d'une perspective utilitariste basée sur les services écosystémiques et d'une perspective holistique basée sur la notion de « Terre Mère ».

2. Nous nous sommes notamment inspirés des critères de (Bagstad et al., 2013 ; Thievent, 2015).

\section{RÉSUMÉS}

Certaines entreprises développent, testent, promeuvent des outils d'évaluation de la biodiversité et des services écosystémiques comme aide à la décision. L'efficacité et les apports concrets de ces outils restent cependant à démontrer. Dix-huit outils recommandés aux entreprises sont évalués sur la base de trois dimensions caractéristiques de l'efficacité des interfaces 
connaissances-décision : la crédibilité, la pertinence et la légitimité. L'analyse quantitative des scores met en évidence des tensions entre ces trois dimensions ainsi que des différences de profils selon : l'implication ou non d'entreprises lors de la conception de l'outil, les types de résultats produits (qualitatifs, monétaires, non monétaires, combinés) et la finalité d'usage de l'outil (diagnostic, pilotage et communication externe).

Businesses are showing some interests in biodiversity evaluation tools. Some companies develop, test and promote these tools as supports for decision-making. The effectiveness and practical effects of these tools still need to be demonstrated. Eighteen tools recommended to business are evaluated on the basis of three dimensions that characterize the effectiveness of knowledgedecision interfaces : credibility, relevance and legitimacy. The quantitative analysis of the scores highlights tradeoffs between these three dimensions as well as different profiles depending on the inclusion of business actors in the conception phase, the type of output (qualitative, monetary, non-monetary, combining monetary and non-monetary) and final use (diagnosis, monitoring and external communication). Thus, these tradeoffs between credibility, relevance and legitimacy should be acknowledged during the tool selection step and consistent with the final use of the results.

\section{INDEX}

Keywords : evaluation tools, biodiversity, ecosystem services, business, credibility, relevance, legitimacy

Mots-clés : outils d'évaluation, biodiversité, services écosystémiques, entreprises, crédibilité, pertinence, légitimité

\section{AUTEURS}

\section{ANASTASIA WOLFF}

Anastasia Wolff est doctorante à l'École des mines de Saint-Étienne (membre de l'UMR 5600 EVS)et ingénieur des Ponts, des Eaux et des Forêts(AgroParisTech). Son doctorat porte sur la contribution de la responsabilité sociétale des entreprises à la conservation de la biodiversité, anastasia.wolff@emse.fr

\section{NATACHA GONDRAN}

Natacha Gondran est maître-assistante à l'institut Henri-Fayol de l'École des mines de SaintÉtienne (membre de l'UMR 5600 EVS). Son habilitation à diriger les recherches porte sur l'évaluation et la représentation des enjeux environnementaux dans une perspective de transitions écologique et énergétique.

\section{CHRISTIAN BRODHAG}

Christian Brodhag est directeur de recherche à l'institut Henri-Fayol de l'École des mines de Saint-Étienne (membre de l'UMR 5600 EVS). Son activité porte sur le développement durable, la RSO et l'innovation. 